\title{
Tooth discolouration and staining: a review of the literature
}

\author{
A. Watts, ' and M. Addy, ${ }^{2}$
}

\begin{abstract}
Objective To carry out an extensive review of the literature on tooth staining with particular regard to some of the more recent literature on the mechanisms of tooth staining involving mouthrinses.

Design Comprehensive review of the literature over four decades.

Conclusions A knowledge of the aetiology of tooth staining is of importance to dental surgeons in order to enable a correct diagnosis to be made when examining a discoloured dentition and allows the dental practitioner to explain to the patient the exact nature of the condition. In some instances, the mechanism of staining may have an effect on the outcome of treatment and influence the treatment options the dentist will be able to offer to patients.
\end{abstract}

$\mathrm{T}_{0}$ he appearance of the dentition is of concern to a large number of people seeking dental treatment and the colour of the teeth is of particular cosmetic importance.

There has been a recent increase in interest in the treatment of tooth staining and discolouration as shown by the large number of tooth whitening agents appearing on the market. Some of these agents are sold as 'over-the-counter' products and have no professional involvement in their application. The correct diagnosis for the cause of discolouration is important as, invariably, it has a profound effect on treatment outcomes. It would seem reasonable, therefore, that dental practitioners have an understanding of the aetiology of tooth discolouration in order to make a diagnosis and enable the appropriate treatment to be carried out.

The purpose of this article is to review the literature on the causes of tooth staining and discolouration as there has been little mention of the aetiology of discolouration since Vogel's review in 1973. ${ }^{1}$

\section{Colour and colour perception}

A basic understanding of the elements of tooth colour is important for many aspects of restorative dentistry. Teeth are typically composed of a number of colours and a gradation of colour occurs in an individual tooth from the gingival margin to the incisal edge of the tooth. The gingival margin often has a darker appearance because of the close approximation of the dentine below the enamel. In most people canine teeth are darker than central and lateral incisors and younger people characteristically have lighter teeth, particularly in

\footnotetext{
${ }^{1}$ Specialist Registrar in Restorative Dentistry ${ }^{2}$ Professor/Honorary Consultant, Division of Restorative Dentistry, Bristol Dental Hospital and School, Lower Maudlin Street, Bristol BS1 2LY

Correspondence to: $M$. Addy

email:Martin.Addy@bristol.ac.uk

REFEREED PAPER

Received 01.06.99; Accepted 20.03.00

(C) British Dental Journal 2001; 190: 309-316
}

the primary dentition. Teeth become darker as a physiological age change, this may be partly caused by the laying down of secondary dentine, incorporation of extrinsic stains and gradual wear of enamel allowing a greater influence on colour of the underlying dentine. Also, and to be discussed further, toothwear and gingival recession can directly or indirectly affect tooth colour.

The science of colour is important in dentistry with regard to colour perception and description, and can be improved with training. ${ }^{2}$ The viewing conditions are extremely important and variables such as the light source, time of day, surrounding conditions and the angle the tooth is viewed from affect the apparent tooth colour. Light is composed of differing wavelengths and the same tooth viewed under different conditions will exhibit a different colour, a phenomenon known as metamerism.

In judging tooth colour it is best if the light source used is standardised to reduce the effects of metamerism. It is not uncommon to find three sources of light in a dental surgery; ${ }^{3}$ natural, fluorescent and incandescent. Particular light sources are known to have an effect at characteristic ends of the spectrum. For instance, incandescent light will accentuate the red-yellow end of the spectrum and weaken the blue end. Conversely, a fluorescent light source has more energy towards the blue-green end of the spectrum and accentuates these colours accordingly. Specially developed colour-corrected lights are available which help to reduce the effects of metamerism, by providing even colour distribution. Natural sunlight varies in its colour, at noon the sky appears blue with minimal atmosphere to penetrate. Early morning and late evening sunlight has a red-orange tinge as the shorter wavelength blue light is scattered by the atmosphere and only red and orange rays penetrate.

The aesthetic aspects of tooth colour are difficult to quantify and colour perception is highly subjective and prone to individual variation. Disagreement between dentists in shade matching the same tooth has been documented by Culpepper, ${ }^{4}$ not only between dentists, but also the same dentist on different occasions.

Colour can be described according to the Munsell terms of hue, value and chroma. ${ }^{5}$ Hue is the descriptive term to enable one to distinguish between different families of colour, for example reds, blues and greens. Value is the relative lightness and darkness of a colour on a scale from black to white. Chroma is the degree of colour saturation and describes the strength of a colour as it changes, for example, from pink to crimson. Miller and co-workers have suggested the addition of a fourth dimension to this three dimensional colour system, in the form of opacity/translucency. ${ }^{3}$

\section{Classification of tooth discolouration ${ }^{6}$}

The coronal portion of the tooth consists of enamel, dentine and pulp. Any change to these structures is likely to cause an alteration in the outward appearance of the tooth caused by its light transmitting and reflecting properties. The appearance of tooth colour is dependent on the quality of the reflected light and is also, as a consequence, dependent on the incident light. 
Historically, tooth discolouration has been classified according to the location of the stain, which may be either intrinsic or extrinsic. It may also be of merit to consider a further category of internalised stain or discolouration. ${ }^{7}$

\section{Intrinsic discolouration}

Intrinsic discolouration occurs following a change to the structural composition or thickness of the dental hard tissues. The normal colour of teeth is determined by the blue, green and pink tints of the enamel and is reinforced by the yellow through to brown shades of dentine beneath. A number of metabolic diseases and systemic factors are known to affect the developing dentition and cause discolouration as a consequence. Local factors such as injury are also recognised.

1. Alkaptonuria

2. Congenital erythropoietic porphyria

3. Congenital hyperbilirubinaemia

4. Amelogenesis imperfecta

5. Dentinogenesis imperfecta

6. Tetracycline staining

7. Fluorosis

8. Enamel hypoplasia

9. Pulpal haemorrhagic products

10. Root resorption

11. Ageing

\section{Extrinsic discolouration}

Extrinsic discolouration is outside the tooth substance and lies on the tooth surface or in the acquired pellicle. The origin of the stain may be:

\section{Metallic \\ 2. Non-metallic}

\section{Internalised discolouration}

Internalised discolouration is the incorporation of extrinsic stain within the tooth substance following dental development. It occurs in enamel defects and in the porous surface of exposed dentine. The routes by which pigments may become internalised are:

1. Developmental defects

2. Acquired defects
a) Tooth wear and gingival recession
b) Dental caries
c) Restorative materials

\section{The mechanisms of tooth discolouration}

\section{Intrinsic tooth discolouration}

The formation of intrinsically discoloured teeth occurs during tooth development and results in an alteration of the light transmitting properties of the tooth structure. As mentioned in the classification section, there are a number of metabolic disorders which affect the dentition during its formation, unlike the inherited disorders in which only the hard tissue forming at the time may be involved. These disorders will now be discussed individually.

1. Alkaptonuria: This inborn error of metabolism results in incomplete metabolism of tyrosine and phenylalanine, which promotes the build up of homogentisic acid. This affects the permanent dentition by causing a brown discolouration. ${ }^{8}$

2. Congenital erythropoietic porphyria: This is a rare, recessive, autosomal, metabolic disorder in which there is an error in porphyrin metabolism leading to the accumulation of porphyrins in bone marrow, red blood cells, urine, faeces and teeth. A red-brown discolouration of the teeth is the result and the affected teeth show a red fluorescence under ultra-violet light. ${ }^{9}$ King George III was said to have suffered with acute intermittent porphyria but with the later onset of

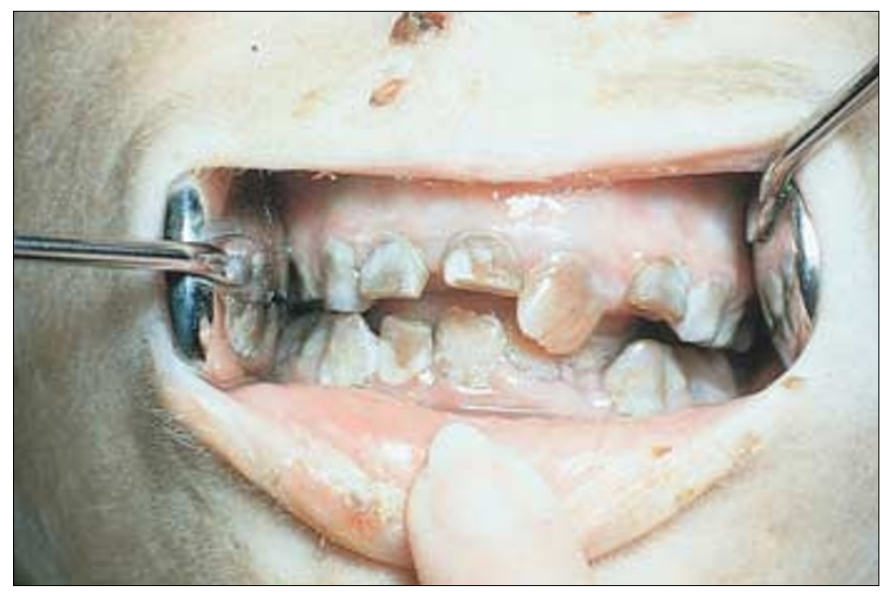

Fig. I Congenital erythropoietic porphyria

this disorder his teeth are unlikely to have been affected (Fig. 1).

3. Congenital hyperbilirubinaemia: The breakdown products of haemolysis will cause a yellow-green discolouration. Mild neonatal jaundice is relatively common, but in rhesus incompatibility massive haemolysis will lead to deposition of bile pigments in the calcifying dental hard tissues, particularly at the neonatal line. ${ }^{10,11}$

4. Amelogenesis imperfecta: In this hereditary condition, enamel formation is disturbed with regard to mineralization or matrix formation and is classified accordingly. There are 14 different subtypes, ${ }^{12}$ the majority are inherited as an autosomal dominant or $\mathrm{x}$-linked trait with varying degrees of expressivity. ${ }^{13,14}$ The appearance depends upon the type of amelogenesis imperfecta, varying from the relatively mild hypomature 'snow-capped' enamel to the more severe hereditary hypoplasia with thin, hard enamel which has a yellow to yellow-brown appearance (Fig. 2).

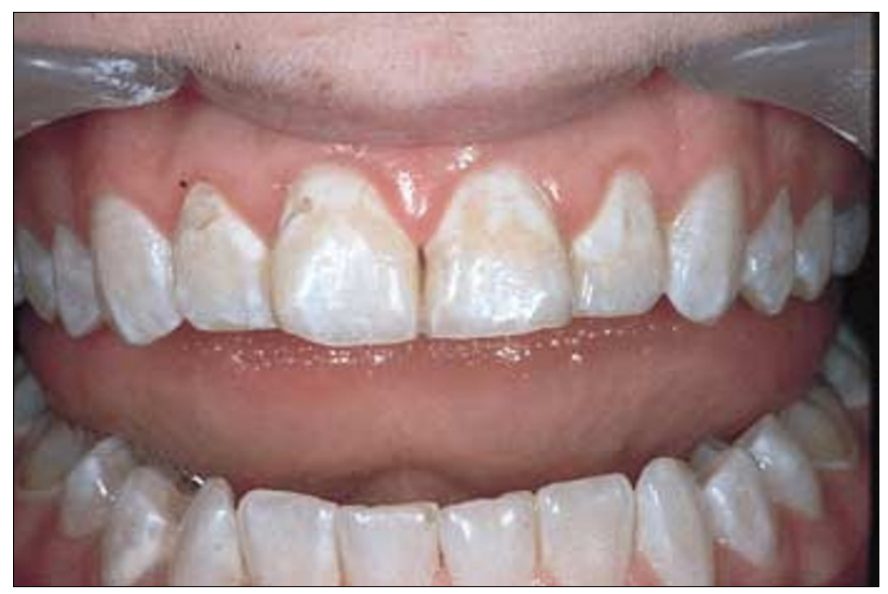

Fig. 2 Amelogenesis Imperfecta

5. Systemic syndromes: Defects in enamel formation may also occur in a number of systemically involved clinical syndromes such as Vitamin D dependent rickets, epidermolysis bullosa and pseudohypoparathyroidism. Barabas ${ }^{15}$ has reported areas of hypoplastic enamel, irregularities in the region of the amelo-dentinal and the cemento-dentinal junctions in Ehlers-Danlos Syndrome. In epidermolysis bullosa there is pitting of the enamel possibly caused by vesiculation of the ameloblast layer. However, the effect of these conditions depends on disease activity during the development of the dentition and is usually a minor element.

6. Dentinogenesis imperfecta: Dentine defects may occur genetically or through environmental influences. ${ }^{16}$ The genetically determined dentine defects may be in isolation or associated with a systemic disorder. The main condition related to the dentine alone 
is Dentinogenesis imperfecta II (hereditary opalescent dentine). Both dentitions are affected, the primary dentition usually more severely so. The teeth are usually bluish or brown in colour, and demonstrate opalescence on transillumination. The pulp chambers often become obliterated and the dentine undergoes rapid wear, once the enamel has chipped away, to expose the amelo-dentinal junction. Once the dentine is exposed, teeth rapidly show brown discolouration, presumably by absorption of chromogens into the porous dentine (Fig 3).

Dentinogenesis imperfecta I (associated with osteogenesis imperfecta, a mixed connective tissue disorder of type I collagen) may show bone fragility and deformity with blue sclera, lax joints and opalescent dentine. The inheritance may be dominant or recessive, the recessive being more severe and often fatal in early life. Opalescent teeth are more common in the dominant inheritance pattern, the primary teeth bear a strong resemblance to the teeth in Dentinogenesis imperfecta type I whereas the appearance of the secondary dentition is much more variable. The enamel is much less prone to fracture, the pulp chamber is seldom occluded by dentine (this may help to radiographically differentiate between types I and II), and the overall prognosis for the dentition is improved. ${ }^{17}$

A third type of Dentinogenesis imperfecta (type III, brandywine isolate hereditary opalescent dentine) was described by Wiktop. ${ }^{17}$ In this condition, the teeth may be outwardly similar to both types I and II of Dentinogenesis imperfecta; however, multiple pulpal exposures occur in the primary dentition. Radiographically, the teeth may take on the appearance of 'shell teeth' as dentine production ceases after the mantle layer has formed. This type of Dentinogenesis imperfecta is thought to be related more closely to type II.

7. Dentinal dysplasias: Shields ${ }^{18}$ reclassified the inherited dentine defects in a review of the literature in 1973 and introduced the term dentinal dysplasias. This reclassification allows separation of the inherited types of dentine defects from Dentinogenesis imperfecta, with which they are often confused.

In type I dentine dysplasia the primary and secondary dentition are of normal shape and form but may have an amber translucency. Radiographically the teeth have short roots with conical apical constrictions. The pulp is commonly obliterated in the primary dentition, leaving only a crescentic pulpal remnant in the adult dentition parallel to the cemento-enamel junction. There are characteristic periapical radiolucencies in many, otherwise healthy, teeth. The

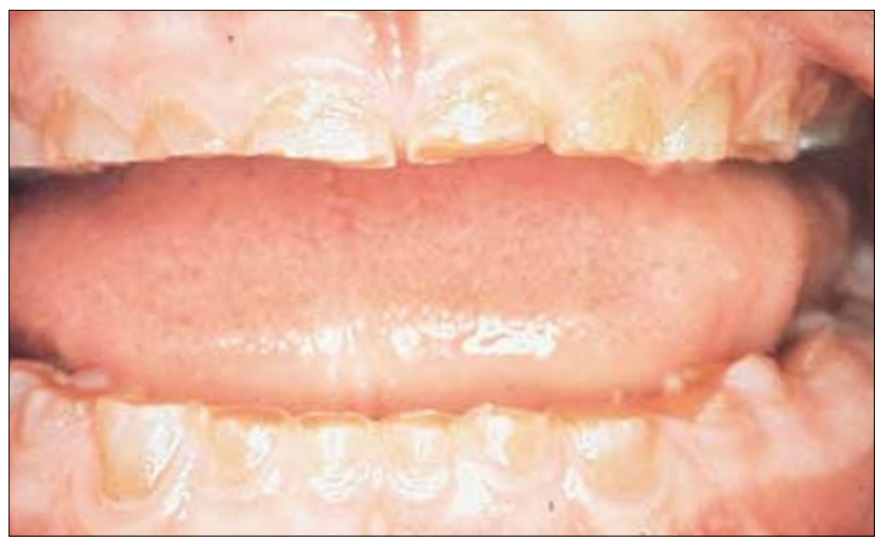

Fig. 3 Dentinogenesis imperfecta

condition is inherited as an autosomal dominant trait

Type II dentine dysplasia is described through a small number of case reports in Shields ${ }^{18}$ review, the main characteristic is that of a thistle-shaped pulp chamber with numerous pulp stones. A brown discolouration of the teeth was mentioned in two of the literature reports cited.

8. Tetracycline staining: Systemic administration of tetracyclines during development is associated with deposition of tetracycline within bone and the dental hard tissues. ${ }^{19,20}$ Urist and Ibsen ${ }^{21}$ suggested that tetracycline and its homologues have the ability to form complexes with calcium ions on the surface of hydroxy apatite crystals within bone and dental tissues. Dentine has been shown to be more heavily stained than enamel. ${ }^{19}$ Tetracycline is able to cross the placental barrier and should be avoided from 29 weeks in utero until full term to prevent incorporation into the dental tissues. Since the permanent teeth continue to develop in the infant and young child until 12 years of age, tetracycline administration should be avoided in children below this age and in breast-feeding and expectant mothers. ${ }^{22}$ The most critical time to avoid the administration of tetracycline for the deciduous dentition is 4 months in utero to 5 months post-partum, with regard to the incisor and canine teeth. In the permanent dentition, for the incisor and canine teeth, this period is from 4 months post-partum to approximately 7 years of age. ${ }^{22}$ The colour changes involved

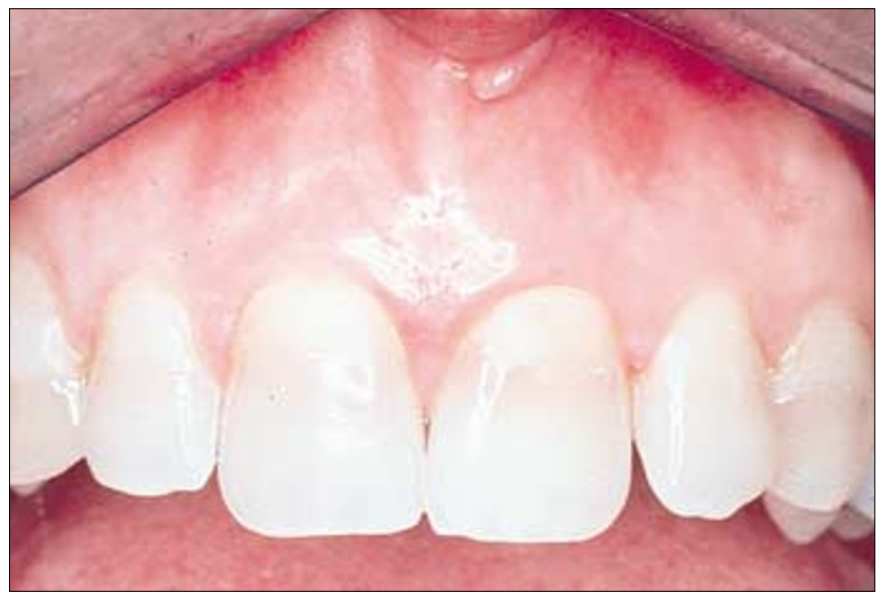

Fig. 4 Tetracycline staining

depend upon the precise medication used, the dosage and the period of time over which the medication was given. Teeth affected by tetracycline have a yellowish or brown-grey appearance which is worse on eruption and diminishes with time. Exposure to light changes the colour to brown, the anterior teeth are particularly susceptible to light induced colour changes. The various analogues of tetracycline produce different colour changes, for instance chlortetracycline produces a slate grey colour and oxytetracycline causes a creamy discolouration. ${ }^{23,24}$ Since tetracycline fluoresces under ultraviolet light so do affected teeth, giving off a bright yellow colour. There have been recent reports of adults experiencing change in tooth colour with the use of long term tetracycline therapy. ${ }^{25}$ Minocycline, a synthetic compound of tetracycline antibiotics, is also implicated in causing discolouration in an adult patient, following its long term use for treatment of acne. ${ }^{26,27}$ This phenomenon was described in a single case report in the literature by Cale et al. ${ }^{28}$ When the appearance of the dentine had altered following the long-term use of minocycline for acne, it was postulated that calcium-minocycline complexes were deposited in the dentine (Fig. 4).

9. Fluorosis: The association between fluoride intake and its effect on enamel was noted by Dean as long ago as $1932 .{ }^{29}$ This may arise endemically from naturally occurring water supplies or from fluoride delivered in mouthrinses, tablets or toothpastes as a supplement. The severity is related to age and dose, with the primary and secondary dentitions both being affected in endemic fluorosis. Birdsong-Whitford et al. ${ }^{30}$ gave evidence for the possible increased affect of fluorosis at altitude with their work on rodents. The enamel is often affected and may vary from areas of flecking to diffuse opacious mottling, whilst the colour of the enamel ranges from chalky 
white to a dark brown/black appearance. The brown/black discolouration is post-eruptive and probably caused by the internalisation of extrinsic stain into the porous enamel..$^{31}$

These features are often described as being pathognomonic of fluorosis, but care should be taken not to confuse the condition with the hypomaturation type of ameolgenesis imperfecta. ${ }^{32}$ Fluoride only causes fluorosis in concentrations of greater than $1 \mathrm{ppm}$ in drinking water and is not distinguishable, clinically or histologically, from any

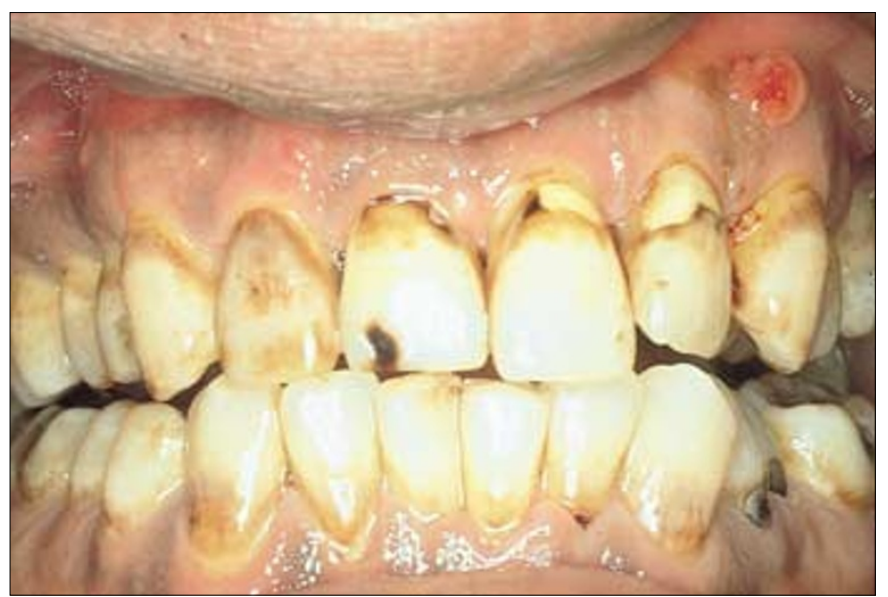

Fig. 5 Dental fluorosis

other type of hypoplastic or hypomineralized enamel (Fig. 5).

10. Enamel hypoplasia: This condition may be localised or generalised. The most common localised cause of enamel hypoplasia is likely to occur following trauma or infection in the primary dentition. ${ }^{33}$ Such localised damage to the tooth-germ will often produce a hypoplastic enamel defect, which can be related chronologically to the injury.

Disturbance of the developing tooth germ may occur in a large number of foetal or maternal conditions eg maternal vitamin $D$ deficiency, rubella infection, drug intake during pregnancy and in paediatric hypocalcaemic conditions. ${ }^{34}$ Such defects will be chronologically laid down in the teeth depending on the state of development at the time of interference, the effect is directly related to the degree of systemic upset. There may be pitting or grooving which predisposes to extrinsic staining of the enamel in the region of tooth disturbed, often then becoming internalised (Fig. 6).

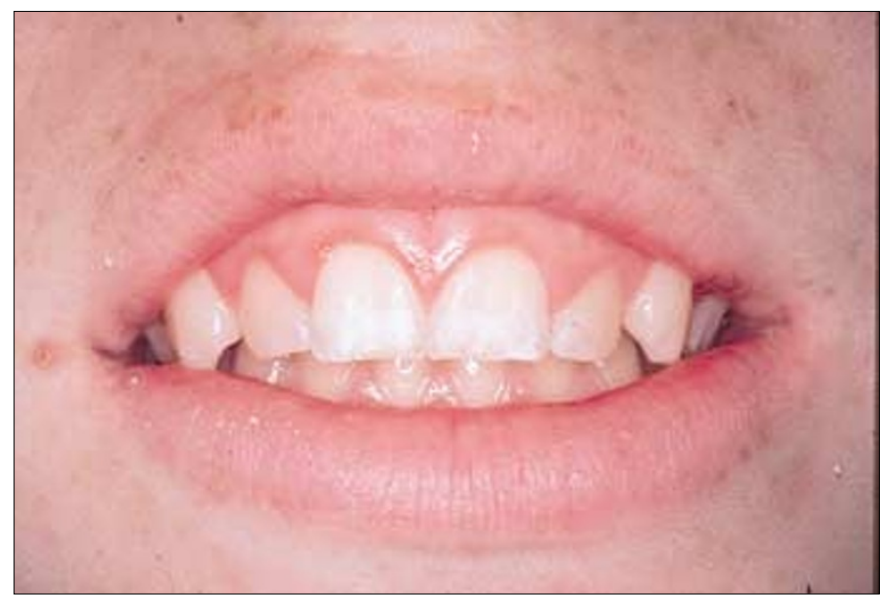

Fig. 6 Localised enamel hypoplasia on both upper central incisors

11. Pulpal haemorrhagic products: The discolouration of teeth following severe trauma was considered to be caused by pulpal haemorrhage. Haemolysis of the red blood cells would follow and release the haem group to combine with the putrefying pulpal tis- sue to form black iron sulphide. Grossman asserted in 1943 that the depth of dentinal penetration determines the degree of discolouration; ${ }^{35}$ there was little if any scientific investigation of this hypothesis. In vitro studies have recently shown that the major cause of discolouration of non-infected traumatised teeth is the accumulation of the haemoglobin molecule or other haematin molecules. In the absence of infection, the release of iron from the protoporphyrin ring is unlikely. This greater understanding of the nature of tooth staining following trauma to teeth may be of importance if the manufacture of bleaching agents, with specific activity, becomes possible. For instance, with further analysis it may become possible to develop a bleaching agent for use on teeth stained specifically by blood pigments. ${ }^{36}$ Incidentally, it has been shown that the pinkish hue seen initially after trauma may disappear in 2 to 3 months if the tooth becomes revascularised (Fig. 7). ${ }^{37}$

Post-mortem changes in tooth colour have been well documented in the literature. A purple-pink discolouration has been noted; Whittaker states that this is not seen in corpses less than 4 weeks old. ${ }^{38}$ Other authors have suggested that the cause of death has an influence on the discolouration, it being more noticeable in carbon monoxide poisoning and drowning. Simpson felt the attitude of the body at the time of death also had an effect on the degree of discolouration in a fashion similar to the 'livid stain' of a post mortem dependent part. ${ }^{39}$ This phenomenon is not of significance

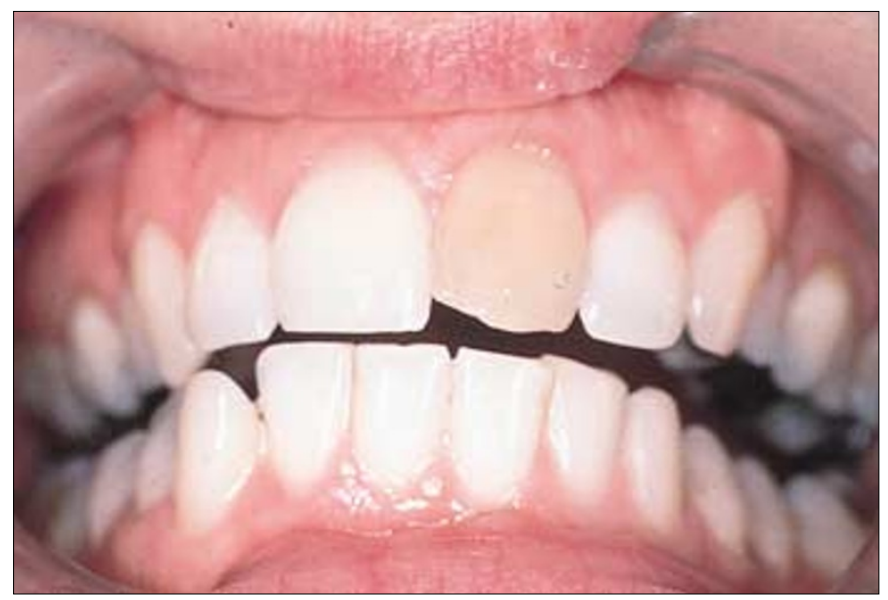

Fig. 7 Haemorrhagic products in a non-vital central

in determining the time of death.

12. Root resorption: Root resorption is often clinically asymptomatic, however, occasionally the initial presenting feature is a pink appearance at the amelo-cemental junction. Root resorption always begins at the root surface, either from the pulpal or periodontal aspect, as internal or external root resorption respectively. It can be difficult to locate a resorptive cavity on radiograph until it reaches a certain size (Fig. 8). ${ }^{40}$

13. Ageing: The natural laying down of secondary dentine affects the light-transmitting properties of teeth resulting in a gradual darkening of teeth with age.

\section{Extrinsic discolouration}

The causes of extrinsic staining can be divided into two categories; those compounds which are incorporated into the pellicle and produce a stain as a result of their basic colour, and those which lead to staining caused by chemical interaction at the tooth surface.

Direct staining has a multi-factorial aetiology with chromogens derived from dietary sources or habitually placed in the mouth. These organic chromogens are taken up by the pellicle and the colour imparted is determined by the natural colour of the chromogen. Tobacco smoking and chewing are known to cause staining, 
as are particular beverages such as tea and coffee. The colour seen on the tooth is thought to be derived from polyphenolic compounds which provide the colour in food. ${ }^{41}$

Indirect extrinsic tooth staining is associated with cationic antiseptics and metal salts. The agent is without colour or a different colour from the stain produced on the tooth surface. Interest in the mechanisms of extrinsic tooth staining was rekindled in 1971 with the observation by Flotra et al., ${ }^{42}$ that tooth staining increases with the use of chlorhexidine.

Extrinsic tooth discolouration has usually been classified according to its origin, whether metallic or non-metallic. ${ }^{43}$

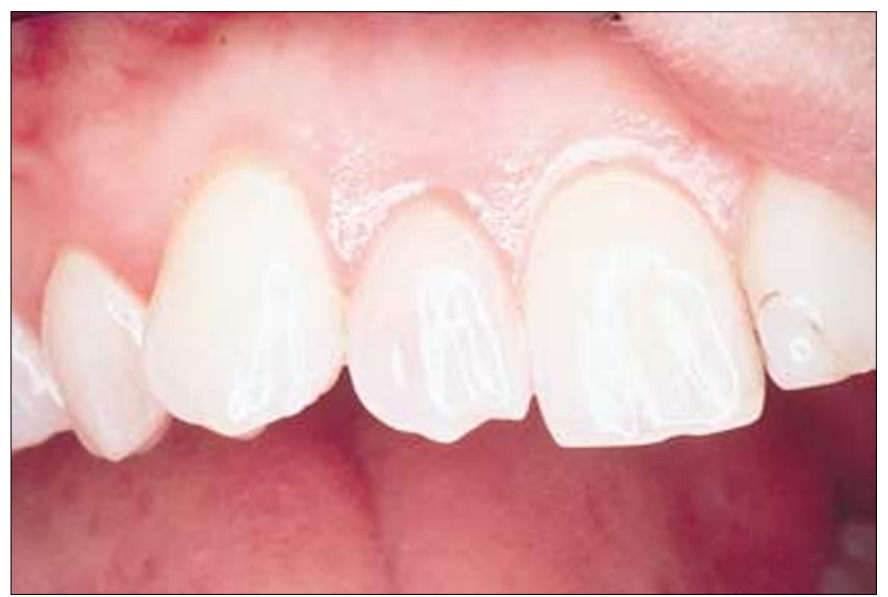

Fig. 8 Pink spot in internal resorbtion

Non-metallic stains: The non-metallic extrinsic stains are adsorped onto tooth surface deposits such as plaque or the acquired pellicle. The possible aetiological agents include dietary components, beverages, tobacco, mouthrinses and other medicaments. Chromogenic bacteria have been cited in children. Particular colours of staining are said to be associated with certain mouths, for instance, green and orange in children with poor oral hygiene and black/brown stains in children with good oral hygiene and low caries experience. ${ }^{44}$ Conclusive evidence for the chromogenic bacterial mechanism has not been forthcoming.

The most convincing evidence for the extrinsic method of tooth staining comes from the differing amount of stain found in a comparison of smokers and non-smokers. ${ }^{45}$ The staining effect of prolonged rinsing with chlorhexidine mouthrinses ${ }^{46}$ and quarternary ammonium compounds used in mouthrinses ${ }^{47}$ is of considerable interest to the dental profession.

Metallic stains: Extrinsic staining of teeth may be associated with occupational exposure to metallic salts and with a number of medicines containing metal salts. ${ }^{46}$ The characteristic black staining of teeth in people using iron supplements and iron foundry workers ${ }^{48}$ is well documented. Copper causes a green stain in mouthrinses containing copper salts ${ }^{49}$ and in workers in contact with the metal in industrial circumstances. ${ }^{50} \mathrm{~A}$ number of other metals have associated colours such as potassium permangenate producing a violet to black colour ${ }^{1}$ when used in mouthrinses; silver nitrate salt used in dentistry causes a grey colour, ${ }^{50}$ and stannous fluoride causes a golden brown discolouration. ${ }^{51}$ It was previously thought that the mechanism of stain production was related to the production of the sulphide salt of the particular metal involved. ${ }^{52}$ This is perhaps not surprising since the extrinsic stain coincided with the colour of the sulphide of the metal concerned. However, those proposing the hypothesis appeared not to consider the complexity of the chemical process necessary to produce a metal sulphide.

As mentioned earlier the interest aroused by the staining noted with use of chlorhexidine mouthrinse has prompted renewed interest in the mechanism of stain formation. For this reason most of the research into stain formation has been carried out on chlorhexidine, although there are other antiseptics which cause staining to a lesser extent and the mechanism proposed could be applicable to staining found with polyvalent metals. The characteristic staining of the tongue and teeth noted by Flotra and co-workers in $1971^{42}$ is not peculiar to chlorhexidine, it has been reported in other cationic antiseptics, ${ }^{53}$ the essential oil/phenolic mouthrinse 'Listerine ${ }^{52}$ and following prolonged use of delmopinol mouthrinses. ${ }^{53}$ There is great individual variation in the degree of staining from person to person, this makes explanation more difficult as it may be caused by intrinsic factors, differences in extrinsic factors or both. No longer accepted theories of stain formation with chlorhexidine include breakdown of chlorhexidine in the oral cavity to form parachloraniline ${ }^{54}$ and also that chlorhexidine may reduce bacterial activity such that partly metabolised sugars were broken down and then degraded over time to produce brown-coloured compounds. ${ }^{55}$ Most recent debate has centred around three possible mechanisms. ${ }^{7}$

Non-enzymatic browning reactions: Berk suggested that the protein and carbohydrate in the acquired pellicle could undergo a series of condensation and polymerisation reactions leading to discolouration of the acquired pellicle. ${ }^{57}$ Chlorhexidine may accelerate formation of the acquired pellicle ${ }^{58}$ and also catalyze steps in the Maillard reaction. Observation of furfurals, intermediate products in Maillard reactions, in brown-discoloured pellicle has leant support to the theory, ${ }^{59}$ but the evidence is inconclusive. ${ }^{60}$ Moreover, these authors did not consider at all the same staining phenomenon observed with the numerous other antiseptics.

The formation of the pigmented sulphides of iron and tin: this theory suggests that chlorhexidine denatures the acquired pellicle to expose sulphur radicals. The exposed radicals would then be able to react with the metal ions to form the metal sulphide. Warner and coworkers ${ }^{61}$ have shown increased levels of iron in chlorhexidine treated individuals compared with water controls, no evidence was shown for tin. They then went on to conclude that the chromophore was not a sulphide, ${ }^{61}$ but a sulphur containing organic compound and metal ion complex and that chlorhexidine promoted the deposition of sulphate proteins. However, somewhat anomalously although the amount of stain and iron levels were increased, the levels of sulphide were reduced. Studies in vitro ${ }^{62}$ have contradicted aspects of the metal sulphide/denaturation theory. ${ }^{54}$ For instance, dietary staining of chlorhexidine treated tooth substance and acrylic occurred in the absence of salivary pellicle. More importantly pellicle coated surfaces exposed to protein denaturants or chlorhexidine did not stain when subsequently exposed to salts of iron and tin. Staining of saliva-coated tooth and acrylic occurred only when the chlorhexidine treatment was followed by a dietary chromogen such as tea. This has to some extent been replicated in vivo, ${ }^{54}$ where reciprocal rinsing with chlorhexidine and iron sulphate produced no staining in volunteers who abstained from food and beverages. However, chlorhexidine or iron sulphate followed by tea rinse produced immediately the characteristic brown and black discolouration of the teeth and tongue reported for chlorhexidine and iron respectively.

Precipitation of dietary chromogens by chlorhexidine: Plaque inhibition is dependent upon adsorption of chlorhexidine onto the tooth surface. ${ }^{55}$ Davies et al. suggested that locally adsorped chlorhexidine complexed with ions from the oral environment and showed this in vitro with the colour produced between chlorhexidine and food dyes. ${ }^{56}$ Following this observation, in vitro and in vivo experiments showed that chlorhexidine and other antiseptics known to cause staining in vivo could bind dietary chromogens to surfaces to produce staining. ${ }^{57-60}$ One objection to the dietary chromogen theory was that there is no known correlation between chromogenic staining and dietary consumption of beverages. ${ }^{69}$ However, tea and coffee and red wine are not the only drinks to contain chromogenic polyphenols ${ }^{70}$ capable of interacting with 
chlorhexidine or polyvalent metal ions. Thus, the fact that staining can be produced in rabbits and dogs, which do not usually imbibe human beverages, can be explained by the presence of other polyphenols within the diet which are able to interact with chlorhexidine. Nevertheless, it was of interest to note that Leonard et al. demonstrated that staining was exaggerated in beagle dogs when tea and coffee was provided in conjunction with chlorhexidine rinses. ${ }^{71}$

Most evidence indicates that the likely cause of staining is the precipitation of anionic dietary chromogens onto adsorped cations. Thus, polyphenols found in dietary substances, being anionic, are able to react with cations adsorped to surfaces such as the cationic antiseptics or polyvalent metal ions to produce staining. ${ }^{7}$ The difference in the potential of various cationic antiseptics to produce staining in vivo can be explained by their differing substantivity, which is consistent with the dietary aetiology. The apparent individual variation in staining noted in particular with chlorhexidine is of interest. It is worthy of note that from the diet controlled studies this variation can not be explained solely as a difference in the quantities of chromogenic agents in any one individual's diet even though abstinence from tea, coffee or red wine virtually eliminates staining from everyone. Clearly differences do exist in the propensity of individuals to produce stain and it is worthy of further investigation as it would be relevant to the need to use cosmetic tooth whitening products. There is no evidence to show that chlorhexidine is any less effective in people with a low susceptibility to staining.

\section{Internalised discolouration}

The stains taken up into the body of enamel or dentine are the same as those causing extrinsic tooth discolouration, including in particular dietary chromogens and the by-products of tobacco smoking. Dental defects permitting the entry of chromogenic material can be classified under the headings of 'developmental and acquired'.

1. Developmental defects: The most important defects are considered under the 'intrinsic tooth discolouration' section of this review.

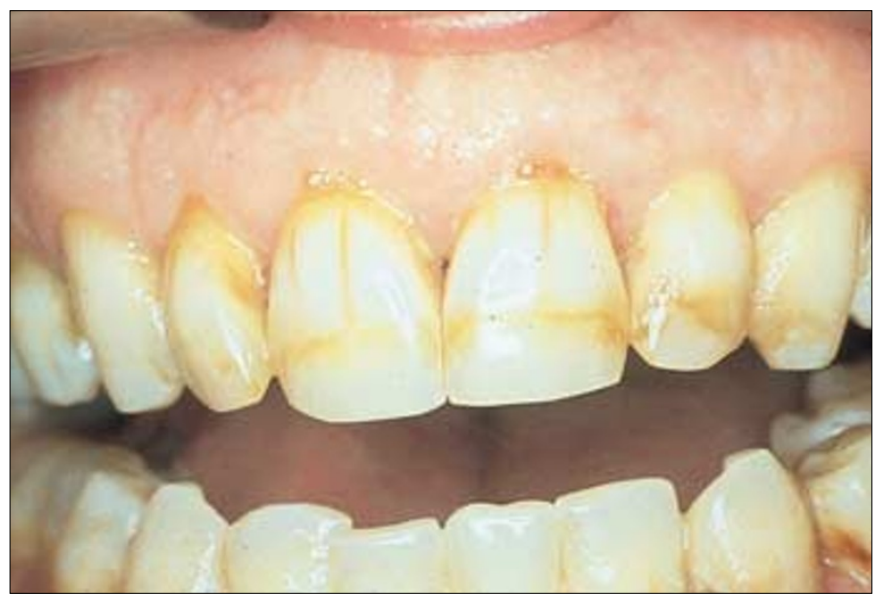

Fig. 9 Internalised stain in enamel cracks

As described these developmental defects create their own colour change in the tooth caused by influences on light transmission through the dentine and enamel. Post-eruptively, however, either caused by increased enamel porosity, or the presence of enamel defects, extrinsic stains can penetrate into the enamel. Such examples would include fluorosis and other enamel conditions resulting in enamel hypoplasia or hypocalcification. Alternatively, developmental defects may expose dentine either directly or later caused by early loss of enamel as in dentinogenesis imperfecta. Chromogens are then able to enter the dentine directly or facilitated almost certainly by the tubule system.
2. Acquired defects: Wear and tear, and disease of the teeth and supporting tissues occur throughout life, all of which can lead directly or indirectly to tooth discolouration. Additionally, repairs on restorations of teeth can influence the colour of teeth(Fig. 9).

a) Tooth wear and gingival recession: Both conditions appear to have multifactorial aetiologies ${ }^{72}$ but to date are poorly understood, there being limited scientific research on the topics. Tooth wear is usually considered to be a progressive loss of enamel and dentine due to erosion, abrasion and attrition. As enamel thins the teeth become darker as the colour of dentine becomes more apparent. Once dentine is exposed the potential of chromogens to enter the body of the tooth is increased. Physical trauma can also result in bulk loss of enamel or enamel cracks, both of which facilitate internalisation of extrinsic stains. Although tooth wear occurs at the cervical area of teeth, where enamel is most thin, exposure of dentine is more likely caused by gingival recession. Again, the net result is dentine exposure and the increased potential for the uptake of chromogens into the tooth (Fig. 10).

b) Dental caries: The various stages of the carious process can be recognised by changes in colour as the disease progresses. For

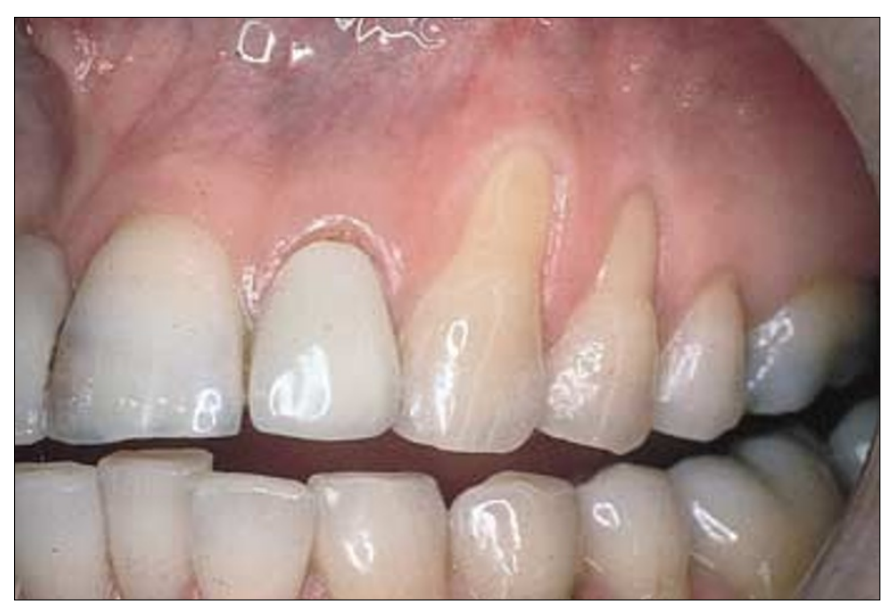

Fig. 10 Gingival recession with dentine discolouration

instance, the initial lesion is characterised by an opaque, white spot. The white spot lesion differs in colour from the adjacent enamel by virtue of its increased porosity and the effect this has on the refractive index. ${ }^{73}$ Enamel has a Refractive Index of 1.62, compared with 1.33 for water and 1.0 for air. Air-drying removes water from the pores in partially demineralized enamel leaving air and makes the 'white spot lesion' conspicuous by the alteration in its light transmitting properties. The hard, arrested lesion is black ${ }^{73}$ having picked up stain from exogenous sources. Early investigation into the change in colour with the carious process centred around the amino-acids released during proteolysis, as a result of the proteolysis-chelation theory of cavity formation. ${ }^{74}$

The sugar-protein reaction is also known as the Maillard reaction or non-enzymatic browning. The reaction is of consequence to the food industry as it results in the browning of bread during the baking process. A huge number of products and intermediate chemicals are involved in the Maillard reaction. Indeed, the brown pigment found in carious dentine was crudely isolated and investigated as long ago as 1950 by Driezen and Spies ${ }^{75}$ and shown to have a similar spectroscopic picture to a synthetic Maillard pigment. However, it has proved difficult to distinguish melanin from Maillard pigments using spectroscopy.

A number of studies have suggested melanin as the cause of discolouration in carious teeth. Kleter and coworkers stated that the evidence for this is poor because of the different sites within a carious lesion at which melanin was discovered. ${ }^{76}$ Furthermore, the silver stain used is not sensitive enough to distinguish melanin from 
pigments such as lipofuschins and bile acids. These pigments could form within the carious lesion, although external pigmentation is a further possibility. Uptake of food dyes into carious lesions has been demonstrated in vivo by Kidd et al. ${ }^{77}$ The evidence regarding carious tooth discolouration is inconclusive. Fusayama et al described how discolouration precedes the bacterial penetration of demineralized dentine ${ }^{78}$ thus it seems that the discolouration is caused by compounds diffusing ahead of the bacteria. In the absence of a relationship between the discolouration and either pigmented bacteria or metal ions Kleter et al. have suggested the formation of melanin or lipofuschin, or a Maillard reaction. ${ }^{67}$ As both melanin and lipofuschin require aerobic conditions for their production, it is thought that small aldehydes can react with proteins under anaerobic conditions to cause browning. An alternative explanation or at least a co-staining factor could again be dietary chromogens entering the dentine consequent upon increased porosity through the carious process.

c) Restorative materials including amalgam: Some of the materials used in restorative dental treatment may have an effect on the colour of teeth. Eugenol and phenolic compounds used during root canal therapy contain pigments which may stain dentine. Some of the polyantibiotic pastes used as root canal medicaments may cause a darkening of the root dentine. Clinicians are familiar with the dark grey to black colour of dentine following the removal of a longstanding amalgam restoration. It was previously thought that mercury was penetrating the dentinal tubules and reacting with sulphide ions. Electronmicroscopic studies have shown that this discolouration is caused by the migration of tin into the tubules. ${ }^{79}$

\section{Summary and conclusions}

It can be seen from the foregoing discussion that an understanding of the mechanisms behind tooth staining is of relevance to the general dental practitioner. In the management of patients with tooth discolouration such information is valuable in the decision-making process when considering whether or not to treat a condition, or refer on to a specialist for an opinion or for treatment. Prevention of avoidable causes of tooth staining is important, the general dental practitioner may be able to offer advice to medical colleagues when alternative therapy is available. A grasp of the pathological processes involved in tooth staining can assist in explaining the cause to anxious or concerned parents. Knowledge of the physiological process involved in shade taking will enable dentists to communicate better with technical staff and also involve the patient in the shade taking process. When recommending or prescribing oral care products known to cause staining, it is important to warn patients of such potential side effects. In understanding the mechanism of stain formation associated with cationic antiseptics and metal salts, notably stannous fluoride, advising patients in respect of the more chromogenic dietary fluids may help prevent or limit tooth staining.

1 Vogel R I. Intrinsic and extrinsic discolouration of the dentition. A review. J Oral Med 1975; 30: 99-104.

2 Bergen. Dentists color matching skills. MSc Thesis. University of California Los Angeles, 1975.

3 Miller L. Organising colour in dentistry. J Am Dent Assoc 1987 (special issue) $26 \mathrm{E}-40 \mathrm{E}$.

4 Culpepper W D. A comparative study of shade-matching procedures. JProsthet Dent 1970; 24: 166-173.

5 Munsell A H. A color notation. Batimore: Munsell Color Co, 1981.

6 Pindborg J J. Pathology of the dental hard tissues. p221. Copenhagen: Munksgaard, 1970.

7 Addy M, Moran J. Mechanisms of stain formation on teeth, in particular associated with metal ions and antiseptics. Adv Dent Res 1995; 9: 450-456.

8 Link J. Discolouration of the teeth in alkaptonuria and Parkinsonism. Chron Omaha Dist Dent Soc 1973; 36: 136.

9 Fayle S A, Pollard M A. Congenital erythropietic porphyria-oral manifestations and dental treatment in childhood: a case report. Quintessence Int 1994; 25: 551-554.
10 Watanabe K, Shibata T, Kurosawa T, Morisaki I, Kinehara M, Igarashi S, Arisue M. Bilirubin pigmentation of human teeth caused by hyperbilirubinaemia. J Oral Pathol Med 1999; 28: 128-30.

11 Wiktop C J Jr. The frequency of discoloured teeth showing yellow fluorescence under ultra-violet light. J Oral Ther Pharmacol 1965; 2: 81-87.

12 Winter G B. Anomalies of tooth formation and eruption. In: Welbury R W (ed) Paediatric dentistry. pp266-270. Oxford: Oxford University Press, 1997.

13 Sundell S, Koch G. Hereditary amelogenesis imperfecta: epidemiology and classification in a Swedish child population. Swed Dent J 1985; 9: 157-169.

14 Wright J, Robinson C, Shoe R. Characterisation of the enamel ultra structure and mineral content in hypoplastic amelogenesis imperfecta. Oral Surg Oral Med Oral Pathol 1991; 72: 594-601.

15 Barabas G M. The Ehlers-Danlos syndrome: abnormalities of the enamel, dentine, cement and dental pulp: a histological examination of twenty-four teeth from six patients. Br Dent J 1969; 126: 509-515.

16 Sclare R. Hereditary opalescent dentine (dentinogenesis imperfecta). $\mathrm{Br}$ Dent J 1984; 84: 164-166.

17 Wiktop C J Jr. Amelogenesis imperfecta, Dentinogenesis imperfecta and dentine dysplasia revisited: problems in classifications. J Oral Pathol 1988; 17: 547-553.

18 Shields E D, Bixler D, El-Kafrawy A M. A proposed classification for heritable dentine defects with description of a new entity. Arch Oral Biol 1973; 18: 543-553.

19 Wallman I S, Hilton H B. Teeth pigmented by tetracycline. Lancet 1962; I: 827-829.

20 Weymann J, Porteous J R. Discolouration of the teeth probably due to administration of tetracyclines: A preliminary report. Br Dent J 1962; 113: 51-54.

21 Urist M R, Ibsen $\mathrm{K} \mathrm{H}$. Chemical reactivity of mineralised tissue with oxytetra cycline. Arch Pathol (Chicago) 1963; 76: 484-496.

22 British National Formulary, March 1999; 37: 254-256, BMJ Books: London, UK

23 Moffitt J M, Cooley R O, Olsen N H, Hefferen J J. Prediction of tetracycline induced tooth discolouration. J Am Dent Assoc 1974; 88: 547-552.

24 van der Bijl P, Pitigoi-Aron G. Tetracyclines and calcified tissues. Ann Dent 1995; 54: 69-72.

25 Chiappinelli J A, Walton R E. Tooth discolouration resulting from long term tetracycline therapy: a case report. Quintessence Int 1992; 23: 539-541.

26 Parkins F M, Furnish G, Bernstein M. Minocycline use discolours teeth. JAm Dent Assoc 1992; 123: 87-89.

27 Patel K, Cheshire D, Vance A. Oral and systemic effects of prolonged minocycline therapy. Br Dent J 1998; 185: 560-562.

28 Cale A E, Freedman P D, Lumerman H. Pigmentation of the jawbones and teeth secondary to minocycline $\mathrm{HCl}$ therapy. J Periodontol 1988; 59: 112-114.

29 Dean H T. Chronic endemic dental fluorosis. JAMA 1932; 107: 1269.

30 Birdsong-Whitford N L, Dickinson A, Whitford G M. Effect of haematocrit on plasma F concentration. J Dent Res 1984; 184: Abstract No. 129.

31 Weatherall J A, Robinson C, Hallsworth A S. Changes in the fluoride concentration of the labial surface enamel with age. Caries Res 1972; 6: 312-324.

32 Crawford P J M, Aldred M J. X-linked amelogenesis imperfecta presentation of two kindreds and a review of the literature. Oral Surg Oral Med Oral Pathol 73: 449-455.

33 Nikiforuk G, Fraser D. The etiology of enamel hypoplasia: a unifying concept. J Pediatr 1981; 98: 888-893.

34 Fearne J M, Bryan E M, Elliman A M, Brook A H, Williams D M. Enamel defects in the primary dentition of children born weighing less than $2000 \mathrm{~g}$. Br Dent J 1990; 168: 433-437.

35 Grossman L. Root canal therapy. 2nd ed. pp 347-349. Philadelphia: Lea and Febiger,1943.

36 Marin P D, Bartold P M, Heithersay G S. Tooth discolouration by blood: an in vitro histochemical study. Endod Dent Traumatol 1997; 13: 132-138.

37 Andraesen F M. Transient apical breakdown and its relation to colour and sensibility changes after luxation injuries to teeth. Endod Dent Traumatol 1986; 2: 9-19.

38 Whittaker D K, Thomas V C, Thomas R I M. Post-mortem pigmentation of teeth. Br Dent J 1976; 140: 100-102.

39 Simpson K. Forensic medicine. 7th ed. p9. London: Edward Arnold.

40 Andraesen F M, Sewerin I, Mandel U. Radiographic assessment of simulated root resorption cavities. Endod Dent Traumatol 1987; 3: 21-27.

41 Pearson D. The chemical analysis of foods. 7th ed. pp 1-450 London: Churchill Livingstone, 1976.

42 Flotra A. Side effects of chlorhexidine mouth washes. Scand J Dent Res 1971; 79: 119-125.

43 Gorlin R J, Goldman H M. Environmental pathology of the teeth. In: Thoma's oral pathology. 6th ed. Vol. I, pp 184-192. St Louis: CV Mosby Co, 1971.

44 Theilade J, Slots J, Fejerskov O. The ultrasound of black stain on human 


\section{REVIEW \\ tooth discolouration and staining}

primary teeth. Scand J Dent Res 1973; 81: 528-532.

45 Ness L, Rosekrans D L, Welford J F. An epidemiologic study of factors affecting extrinsic staining of teeth in an English population. Community Dent Oral Epidemiol 1977; 5: 55-60.

46 Addy M, Roberts W R. The use of polymethylmethacrylate to compare the adsoprtion of staining reactions of some cationic antiseptics. J Periodontol $1981 b ; 52: 380-385$.

47 Farmer E D, Lawton F E. Stains and Discoloration of the Teeth. In: Stones oral and dental diseases. 5th ed. pp511-527. Edinburgh and London: ES Livingstone, 1966.

48 Nordbo H, Eriksen H M, Rolla G, Attramadal A, Solheim H. Iron staining of the acquired enamel pellicle after exposure to tannic acid or chlorhexidine. Scand J Dent Res 1982; 90: 117-123.

49 Waerhag M, Gjermo P, Rolla G, Johansen J R. Comparison of the effect of chlorhexidine and $\mathrm{CuSO}_{4}$ on plaque formation and development of gingivitis. J Clin Periodontol 1984; 11: 176-180.

50 Dayan D, Heifferman A, Gorski M, Begleiter A. Tooth discolouration extrinsic and intrinsic factors. Quintessence Int 1983; 2: 195-199.

51 Ellingsen J E, Eriksen H M, Rolla G. Extrinsic dental stain caused by stannous fluoride. Scand J Dent Res 1982; 90: 9-13.

52 Moran J, Addy M, Pal D, Newcombe R. Comparison of phenolic 0.2\% chlorhexidine products on the developoment of plaque and gingivitis. Clin Prev Dent 1991; 13: 31-35.

53 Claydon N, Hunter L, Moran J et al. A 6-month home usage trail of $0.1 \%$ and $0.2 \%$ delmopinol mouthwashes. Effects on plaque, gingivitis, supragingival calculus and tooth staining. J Clin Periodontol 1996; 23 : 220-228.

54 Gjermo P, Rolla G, Arskaug L. Effect of dental plaque formation and some in vitro properties of 12 bisbiguanides. J Periodontal Res 1973; 12: 81-88.

55 Davies R M, Jensen S B, Schiott C R, Loe H. The effect of topical application of chlorhexidine on the bacterial colonization of the teeth and gingiva. J Periodont Res 1970; 5: 96-101.

56 Addy M, Moran J, Griffiths A, Wills-Wood N J. Extrinsic tooth discoloration by metals and chlorhexidine. Surface protein denaturation or dietary precipitation? Br Dent J 1985; 159: 281-285.

57 Berk Z. Non-enzymatic browning. In: Braveman's introduction to the biochemistry of foods. pp 149-167. Amsterdam: Elsevier, 1976.

58 Yates R, Jenkins S, Newcombe R G, Wade W G, Moran J, Addy M. A 6 month home usage trail of $1 \%$ chlorhexidine toothpaste. Effects on plaque, gingivitis, calculus and tooth staining. J Clin Periodontol 1993; 20: $130-138$.

59 Nordbo H. Discolouration of dental pellicle by tannic acid. Acta Odonto Scand 1977; 35: 305-310.

60 Eriksen H M, Nordbo H, Kantanen H, Ellingsen J M. Chemical plaque control and extrinsic tooth discoloration. A review of possible mechanisms. J Clin Periodontol 1985; 12: 345-350.

61 Warner R R, Myers M C, Burns J, Mitra S. Analytical electron microscopy of chlorhexidine induced stain in humans: direct evidence for metal induced stain. J Periodont Res 1993; 28: 255-265.
62 Addy M, Moran J. Extrinsic tooth discoloration by metals and chlorhexidine. Clinical staining produced by chlorhexidine, iron and tea. Br Dent J 1985; 159: 331-334.

63 Jenkins S, Addy M, Wade W. The mechanism of action of chlorhexidine. A study of plaque growth on enamel inserts in vivo. J Clin Periodontol 1988; 15: 415-424.

64 Jensen J E. Binding of dyes to chlorhexidine-treated hydroxyapatite. Scand J Dent Res 1977; 85: 334-340.

65 Addy M, Prayitno S, Taylor L, Cadogen S. An in vitro study of the role of dietary factors in the aetiology of tooth staining associated with the use of chlorhexidine. J Periodont Res 1979; 14: 403-410.

66 Prayitno S, Taylor L, Cadogan S, Addy M. An in vivo study of dietary factors in the aetiology of tooth staining associated with the use of chlorhexidine. $J$ Periodont Res 1979; 14: 411-417.

67 Addy M, Roberts W R. Comparison of the bisbiguanide antiseptics alexidine and chlorhexidine. II: Clinical and in vitro staining properties. J Clin Periodontol 1981a; 8: 220-230.

68 Addy M, Moran J. The formation of stain on acrylic surfaces by the interaction of cationic antiseptic mouthwashes and tea. J Biomed Mater Res 1984; 18: 631-641.

69 Eriksen H M, Nordbo H, Kantanen H, Ellingsen J M. Chemical plaque control and extrinsic tooth discoloration. J Clin Periodontol 1985; 12 345-350.

70 Harler C R. Tea manufacture. pp 13-22. London: Oxford University Press, 1963.

71 Leonard G J, Witt J J, Underwood R A. The enhancement of chlorhexidine stain in dogs. J Dent Res 1980; 59 (Special issue A-D): 27.

72 Smith R G. Gingival recession. Reappraisal of an enigmatic condition and a new index for monitoring. J Clin Periodontol 1997; 24: 201-205.

73 Thylstrup A, Ferjerskov O. Clinical and pathological features of dental caries. In: Textbook of clinical cariology. 2nd ed. pp 130-136. Copenhagen: Munksgaard, 1995.

74 Banting D W. Management of dental caries in the older patient. In: Geriatric dentistry. pp 141-167. Chicago: Mosby Year Book, 1991.

75 Driezen S, Spies T D. A note on the production of a yellow-brown pigment in the organic matrices of noncarious human teeth by oral lactobacilli. Oral Surg Oral Med Oral Pathol 1950; 3: 686-691.

76 Kleter G A, Damen J J M, Buijs M J, Ten Cate J M. Modification of amino acid residues in carious dentin matrix. J Dent Res 1998; 77: 488-495.

77 Kidd E A M, Joyston-Bechal S, Smith M M. Staining of residual caries under freshly packed amalgam restorations exposed to tea/chlorhexidine in vitro. Int Dent J 1990; 40: 219-224.

78 Fusayama T, Okuse K, Hosoda H. Relationship between hardness, discolouration, and microbial invasion in carious dentine. J Dent Res 1996; 45: 1033-1046.

79 Wei S H, Ingram M I. Analysis of the amalgam tooth interface using the electron microprobe. J Dent Res 1969; 48: 317. 